\title{
Relocation of Vulnerability in Neonatal Intensive Care Medicine
}

Citation for published version (APA):

Mesman, J. (2014). Relocation of Vulnerability in Neonatal Intensive Care Medicine. In A. Hommels, J. Mesman, \& W. E. Bijker (Eds.), Vulnerability in Technological Culture: New directions in research and governance. (pp. 71-88). The MIT Press. Inside Technology

https://doi.org/10.7551/mitpress/9209.003.0006

Document status and date:

Published: 01/01/2014

DOI:

10.7551/mitpress/9209.003.0006

Document Version:

Publisher's PDF, also known as Version of record

Document license:

Taverne

Please check the document version of this publication:

- A submitted manuscript is the version of the article upon submission and before peer-review. There can be important differences between the submitted version and the official published version of record.

People interested in the research are advised to contact the author for the final version of the publication, or visit the DOI to the publisher's website.

- The final author version and the galley proof are versions of the publication after peer review.

- The final published version features the final layout of the paper including the volume, issue and page numbers.

Link to publication

\footnotetext{
General rights rights.

- You may freely distribute the URL identifying the publication in the public portal. please follow below link for the End User Agreement:

www.umlib.nl/taverne-license

Take down policy

If you believe that this document breaches copyright please contact us at:

repository@maastrichtuniversity.nl

providing details and we will investigate your claim.
}

Copyright and moral rights for the publications made accessible in the public portal are retained by the authors and/or other copyright owners and it is a condition of accessing publications that users recognise and abide by the legal requirements associated with these

- Users may download and print one copy of any publication from the public portal for the purpose of private study or research.

- You may not further distribute the material or use it for any profit-making activity or commercial gain

If the publication is distributed under the terms of Article $25 \mathrm{fa}$ of the Dutch Copyright Act, indicated by the "Taverne" license above, 
and is targeted It is called "new" inst feudal lords.

ted this out (Gill c; Vasavi, 2008).

vidarbha-for-sale

\section{Relocation of Vulnerability in Neonatal Intensive Care Medicine}

Jessica Mesman

All of us are exposed to various sorts of threats and adverse influences on a daily basis. Our vulnerability is perhaps revealed most vividly in critical care practices such as emergency rooms, operating rooms, and intensive care units. In these places, our helplessness comes to the fore and our sense of control easily breaks down. At the same time, these places were specially designed to serve as strongholds against our finitude and fragility. In these zones of protection, health care practitioners do all they can to prevent further harm and help patients cope with their vulnerability. This chapter explores vulnerability in one particular critical care practice: the Neonatal intensive care unit (NICU) in an American and Dutch hospital. Highlighting the NICU as a contact zone of high-tech medicine and exceptionally fragile human life, the cutting-edge medical interventions performed here provide an exemplary case for studying vulnerability. ${ }^{1}$

The NICU specializes in the care and treatment of newborns. Newborn babies end up here because their lives are seriously at risk on account of complications linked to their delivery, congenital diseases, infections, food intolerance, or premature birth. As such, these places require a high level of specialization and expertise. Considering the fragility of the patient population, the risk of medical errors is substantial and they may have unacceptable and irreversible consequences. In other words, an NICU involves risky practices, and this calls for a maximum level of reliability. It is here that we can study some of the concrete vulnerabilities in the health care system.

This chapter aims to provide an illustration of the diverse and persistent nature of vulnerability. In the NICU practice, different kinds of vulnerabilities coexist, interact, and, as I will demonstrate, even compete in some cases. As such, my argument will illustrate how, when, and where these vulnerabilities complicate the NICU practice and its associated safety work. Moreover, the only outcome of some of the measures to cope with NICU vulnerability is its relocation. Apart from working toward the 
normalization of risks, health care, as I will show, also entails incessant relocation of vulnerability.

If scholars frame vulnerability mostly within an existential biological understanding as an intrinsic property of human existence, the empirical data presented in this chapter suggest that such a view depicts vulnerability inadequately. ${ }^{2} \mathrm{My}$ argument in fact underscores that vulnerability is a relational concept. By concentrating on the actual practices performed in the NICU, the emergent nature of vulnerability becomes visible. This implies there is not one, stable form of vulnerability, nor is there a single standard for what is considered vulnerable. Instead, vulnerability is always the outcome of situated specifics.

To structure the argument of this chapter, I address vulnerability first at the level of the actors, and subsequently at the level of arrangements. Moreover, each of these sections discusses a different theme. In the section "Vulnerable Patients, Parents, and Practitioners," I describe the different shapes of vulnerability (including the positive ones) when I discuss the situation of these actors. Some of the instances covered will already reveal how vulnerability is relocated through space and time. The theme of relocation will be developed further in the third section "The Brittleness of Reliable Arrangements," where I analyze the arrangements of the NICU's tightly knit organization and demonstrate how measures to deal with vulnerabilities are not always successful. Rather than diminishing vulnerability, actors merely move it from one place to another.

\section{Vulnerable Patients, Parents, and Practitioners}

The children in the NICU unit, by definition, are in a life-threatening condition and need special care. After several days or weeks of treatment in this unit, most of them will manage to cope without all the technology, and they will have turned into the babies that their parents had hoped for after all. However, before getting to that stage, a lot needs to be done, while more often than not, the treatment trajectory is complex and risky. Because the trajectory involves a diversity of actors-including patients, their family, doctors, nurses, and their technological equipment-I will not only focus on the fragility of NICU babies, but I will also describe how vulnerability relates to parents, practitioners, and machines in its own way. Thus, I can demonstrate the emergent nature of vulnerability and its diverse shape in detail, including its positive dimension. As I will show next, the emergent character of vulnerability becomes apparent in the ways that the NICU patients acquire meaning on the ward. 
itails incessant

ntial biological $\therefore$ the empirical ts vulnerability ability is a relasformed in the le. This implies single standard always the out-

Inerability first $f$ arrangements. e. In the section be the different en I discuss the iti already reveal e theme of relohe Brittleness of is of the NICU's 10 deal with vulshing vulnerabil-

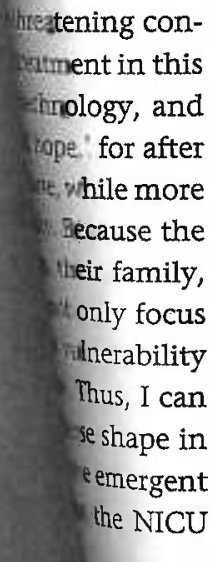

\section{Fragile Patients}

Newborns find themselves in one of their most vulnerable stages of life and are therefore at risk by definition. The NICU automatically admits all children born after a term of less than 32 weeks or with a birth weight under 1,000 grams. ${ }^{3}$ Because the vital functions of premature infants are not well developed yet, they lack the capability to self-regulate their body systems properly, and this may cause a multitude of problems. If, for instance, their immature lungs fail to provide the body with the full amount of oxygen required for survival, the NICU staff immediately has to connect the baby to a respirator for oxygen supply. The baby's circulatory system may also develop problems due to immaturity. The fragility of the blood vessels, in combination with irregular blood pressure, may cause the vessels to be ruptured, while high blood pressure can lead to severe brain damage. And there are many other potential problems: a delicate fluid balance due to a thin skin can bring a premature baby into a state of supercooling and dehydration; kidneys often have trouble functioning; and the infant may simply "forget" to breathe because of the immaturity of the respiratory mechanism in the brain. In response to this, the heart rate can drop rapidly.

As is true in all health care practice, medical interventions can have unintended consequences, no matter how well intended or carefully performed they are. For example, prolonged mechanical breathing with high oxygen concentrations or high air pressure can damage the eyes, cause infection, or damage the fragile lungs of premature babies to such an extent that it reduces the intake capacity to an unacceptable level (Latini et al., 2003). In these cases, it is hard-sometimes even impossible-to reduce the machine's input. Additionally, sedation to calm the intubated baby sometimes leads to addiction and a stomach ulcer. In these cases, the single decision of providing air supply to a prematurely born baby has relocated vulnerability from one place (the immature lungs) to other areas in the body (eyes, lungs, and stomach). Solving one problem (insufficient oxygen intake) generates several new potential problems (eye and lung tissue damage, addiction, and stomach ulcers). This potential relocation of vulnerability due to a medical intervention is an issue that the NICU staff confronts on a daily basis. ${ }^{*}$

Considering the list of possible dangers NICU patients must cope with, providing treatment to them is much like tightrope walking: a minor change in their health status can have dramatic consequences. For this reason, all activity is geared toward stabilizing an NICU baby. This is not only crucial for premature babies, but also for post-operative patients. Take, for instance, Daniel, a baby boy who was born with a major malformation 
of the heart that called for instant intervention. The corrective surgery was complicated and risky. Although Daniel survived the actual surgery, which lasted more than 8 hours, there was still a substantial risk that he would die in the first 48 hours. The instability of his condition made him too vulnerable to be touched, and every transitional moment became a life-threatening event. As the following excerpt from my notes will show, his return to NICU immediately required a most risky transition: taking him out of the reanimation cart and putting him into his incubator:

Now the moment has come to pick him up and put him back into his own bed. The staff do not just have to lift the baby's tiny body, but also the extensive technoalthough all are well prepared, they barely manage to unravel the spaghetti of lines and tubes. Extreme caution is called for, but it also has to be done fast and smoothly. The sooner he is comfortably lying in his incubator, the better. ing so without de-stabilizing Daniel. Everyone is visibly relieved. In following days, Daniel was considered the most vulnerable patient on the con not ward. A "hands-off" policy was established in his case, implyin this body. After three even allowed to wash away the blood and iodine the child: despite several collapses, days the staff began to refer to him as a very strongh the staff continued to be fully he was able to climb out of them each time Although the Daniel a vulnerable child. aware of his serious condition, they no longer cons strength. Likewise, the parents of They interpreted his persistent crying as a sign of sta ling notion that the other children on the NICU began to see Daniel as lining prope and strength. Still, "everything is possible," and he became their symbol seeing him after surgery, his own parents did not dare to touch him for days too vulnerable and not stable let alone take him on their lap. For them he was stil too vulnerible only after nearly enough to be

is the emergent character of vulnerability. Instead of being an intrinsic property, being vulnectives. Although out to be the outcome of specific circumstances and paniel's condition, he was the NICU staff did not deny the seriousness of Der babies on their NICU, he one of their strongest patients. Unst strong enough to cry all day. The staff was born at a mature age and was strong enc characterized him as "strong" only compared to the other ward and his poor condition upon his almerable child. To them, his vulnerahand, considered him an extremely vulnerable chelation to the healthy babies bility emerged as an all-encomparide As such, there is not a single standard that they saw in the outside worle, strong, or safe; rather, the situational for what is considered vulnerable, strong, or safe, rather, the
specifics determine how various actors conceive of vulnerability. 
ive surgery was surgery, which at he would die im too vulnera life-threaten$N$, his return to ing him out of

to his own bed. xtensive technose to unravel the o has to be done bator, the better. ; visibly relieved. le patient on the $t$ nurses were not body. After three several collapses, inued to be fully vulnerable child. ie, the parents of If the notion that Id strength. Still im after surgery, le and not stable only after nearly

haracter of vululnerable turns tives. Although adition, he was their NICU, he 1 day. The staff children on the its, on the other $\mathrm{em}$, his vulneraehealthy babies single standard the situational bility.
Typically, however, vulnerabilities hardly come alone on the NICU. There are multiple vulnerabilities, and they sometimes compete. For instance, some nurses consider a restricted feeding policy in cases of prematurity more appropriate because it avoids the risk of necrotizing enterocolitis, causing portions of the bowel to undergo tissue death due to infection. The medical team claims, by contrast, that an increased caloric intake will make the baby grow, and as such, be more resistant to infections. In this case, the vulnerability of the premature baby's immune system competes with the vulnerability of the baby's bowels. Rather than being a fixed parameter or a well-defined area in NICU practice that everyone agrees on, vulnerability emerges in relation to specific circumstances.

\section{Parents as Second-Order Patients}

Essentially, the treatment trajectory of babies on the NICU is highly unpredictable. No wonder many parents fear a bad outcome, and that may prevent them from bonding with their child. This feeling of separation from their child, in addition to their not having gotten the healthy baby that they had hoped for in the first place, adds to their sense of loss. This triggers a mourning process marked by a variety of reactions, including anger, disbelief, panic, emotional withdrawal, and resignation. In response, the NICU staff tend to refer to parents and children as constituting a single unit of care. Providing support to parents, then, plays a major role in an NICU. Nurses in particular are in charge of giving this support: They inform them about their child's treatment, show them around the NICU, and tell them in everyday language about their child's condition. If physicians have authority over a child's condition, nurses are in charge of dealing with the parents. In this respect, nurses act as "emotional managers" who evaluate and manage parents ${ }^{\prime}$ attachment to their baby (Anspach, 1993, p. 144).

In both the American and the Dutch NICUs I studied, parents are considered as having a psychosocial problem, rather than a medical one, and this problem calls for a psychosocial approach; put differently, they are considered "second-order patients" (Guillemin \& Holmstrom, 1986). In the context of American NICUs, Carson (1996) has pointed to a rise of "socially complex families" due to drug use, teenage parenthood, illiteracy, poverty, AIDS, and aggression. The psychosocial problems that are part of complex family situations may confront staff with new dilemmas and problems about the level of parental understanding of information, who takes part in the decision-making process, and who is capable of bearing the responsibility for the child after its discharge. This is why Carson argues that the 
social worker should play a larger role in the care and aftercare aimed at these more vulnerable families.

A baby's discharge from the hospital will not resolve the problems involved automatically, however. For one thing, many parents of NICU babies continue to perceive their child as medically vulnerable and act accordingly. Several studies show how a high maternal perception of child vulnerability leads to disproportionately high health care utilization, and more important, it also correlates with a worse developmental outcome (Allen et al., 2004; Eiser et al., 2005; Thomasgard, 1998). Parents who nearly lost their child tend to shelter it inordinately; they are alert and take its vulnerability into account with everything they do and plan. But at the same time, they should provide opportunities for their child to experience normal challenges to develop a certain degree of independence (Eiser et al., 2005). If former NICU patients have less opportunity to grow on their own, high parental anxiety also affects their parents. Caring for vulnerable children is considered more stressful. Allen et al. (2004) describe how mothers who perceive their child as vulnerable felt more anxiety and were more depressed, while they were also less optimistic, experienced less satisfaction, and received less social support (p. 271). In other words, vulnerable babies produce vulnerable parents, who in turn are likely to raise vulnerable children.

This particular dynamic involving the parents of NICU babies exemplifies the spatial and temporal relocation of vulnerability. Especially remarkable in this respect is the extensive range of relocation, spatially (from the hospital to the home) and temporally (from babyhood into childhood). However, NICU-instigated vulnerabilities travel far, and not just in the case of parents; as discussed next, physicians have to deal with vulnerabilities that have an impact beyond the hospital walls as well.

\section{Practitioners and Their Vulnerabilities}

Not only are NICU patients and parents vulnerable in their own way; those who take care of them also have their Achilles' heel, if only because life in the NICU is determined by the fluctuations in patients' conditions. The basic task of the NICU staff is to respond adequately to the frequently changing conditions of its patients. As a consequence of the real-time nature of the work, these changes must be dealt with while they are evolving. A complicating factor in this matter is the fact that these changes are not always predictable, let alone unambiguous. By the same token, not all NICU children respond to treatment according to the handbooks, while factual data on their conditions are ambiguous or not yet available. 
aftercare aimed at

ive the problems - parents of NICU ulnerable and act verception of child re utilization, and pmental outcome 198). Parents who $\gamma$ are alert and take Id plan. But at the :hild to experience ıdence (Eiser et al., jow on their own, or vulnerable chilsribe how mothers ty and were more snced less satisfacwords, vulnerable sly to raise vulner-

IU babies exempliEspecially remarkspatially (from the d into childhood). not just in the case vith vulnerabilities

leir own way; those only because life in ts' conditions. The it to the frequently ie of the real-time hile they are evolv$t$ these changes are le same token, not to the handbooks, ir not yet available.
Uncertainty is a characteristic element of medical practice as a whole. In other words, practitioners have to act, even when they have no solid base to act on. Take Julian, a premature baby on the American NICU who unexpectedly entered a serious crisis:

Julian looks terribly pale. The monitor indicates some serious trouble. Until now he was doing so well that this morning his oxygen tube was removed and replaced by a non-invasive form of respiration. Although all day Julian continued to have good blood gas values, he no longer seems able to cope. Instant reconnection is the obvious choice for intervention. But the doctor hesitates: re-intubation is so stressful for Julian that it would dangerously destabilize him. But a glance on the monitor indicates that he cannot do without it.

This raises the issue of which vulnerability should be the indicative factor, the numbers on the monitor or the doctor's experiences with Julian? Re-intubation might be a life-saving intervention for one child, but it can be too stressful and risky for another. The doctor decided to wait two minutes to see if Julian could climb out of his crisis himself before re-intubating him. Thus, he weighed Julian's vulnerability in regard to oxygen intake against his vulnerable cardiac responses to these interventions. In this case, the baby recovered on his own, which solved the dilemma. It suggests that the same condition (low oxygen intake) can be risky and in need of immediate re-intubation in one situation and not in another. This is why many NICU efforts also have an experimental basis: they involve specific attempts to influence the course of events so that a child's condition will improve.

Fortunately, an NICU is a high-tech environment where one will normally find the most advanced machines available. Medical technology thus provides the staff with a major basis for treating and providing safe and reliable care to critically ill newborns. Lined up side by side, the incubators are connected to an array of state-of-the-art medical technologies through all sorts of tubes and cables. These machines, however, are vulnerable in their own way. At some point, they may become inaccurate or even break down. Surprisingly, perhaps, many actors consider older NICU technology to be more reliable than the latest types. The older machines tend to be simpler and less troublesome. The neonatologists and nurses I talked to complained that the more options a device has, the more complex it seems to be, and therefore it is more prone to inaccuracy and false alarms. For this reason, the older models are not replaced immediately when new models arrive. Initially, both are used simultaneously to corroborate and stabilize the new device. If needed, the technology is sent back to its producer, 
while in the meantime, the NICU staff continues to use the old equipment. Manufacturers of the medical technology will also visit the ward regularly to measure the output and performance of their devices. But even when everyone is satisfied by the performance of the technology, the nursing staff continues to corroborate and check them at the beginning of every shift.

In other words, high-tech equipment purchased to make treatment trajectories more secure and precise cannot be integrated automatically as reliable tools. Instead, the staff has to make sure it is reliable. Until then, it is a risky instrument that may well undermine the basis for proper treatment decisions.

Aside from technology settings and outputs, nurses monitor their patients closely. Unlike doctors, nurses spent their whole shift near a baby's incubator. While witnessing its helplessness, they have to come to terms with their own vulnerability as well. Specific characteristics of the NICU practice, such as high turnover of patients and the climate of urgency, help to shield practitioners from confronting this existential vulnerability. But this walling-off becomes more difficult in the case of chronic patients, who sometimes may stay on for months. Because nurses spend a great deal of time with them, they grow familiar with their presence, while their prolonged engagement also tends to foster a more personal contact with the parents. This puts the nurses in a vulnerable position: the stronger the bond, the bigger the loss when the baby dies or is discharged and ready to go home. As one of the nurses in the American neonatology ward pointed out:

You try hard to provide the best possible care to the child. Because you are constantly caring for the same child, you know well how he is doing. No one else follows his medical course as closely. But you also learn to know the child very well in terms of what he likes or dislikes. Of course you grow attached. It's almost like he is one of your own children. And you also become involved with the parents, for you see them almost every day as well. There is a constant balancing between emotional bonding and knowing that one day he will go home, if, at least, all goes well.

Nursing care implies emotional involvement. In some hospitals, staff members deal with this through the organization of their work, whereby they try to strike a balance between continuity and rotation of shifts. For example, nurses in the Dutch NICU that I studied may provide care to the same child for three or four days in a row, but not weeks in a row. This policy is meant to prevent them from becoming emotionally overburdened. This contrasts with the American NICU that I studied, where nurses worked on the basis of primary nursing, meaning that one nurse is 
primarily responsible for the care of a child throughout its entire stay-no matter the length.

Although the system of primary nursing assumes that the nursing staff have good insight into a child's condition, keeping some emotional distance is important as well. Based on their experience, some nurses in the American NICU argued that too much bonding would cloud their professional gaze. In this respect, it is important to note that a strong emotional engagement does not always distort prognostic judgment. Instead it provides a basis for understanding a family's anguish (Benner, Tanner, \& Chesla, 1996). Nor is emotional detachment always a virtue, given that it can blind people's awareness of suffering and lead to needlessly aggressive medical interventions. It is inevitable for nurses, then, to come to terms with their attachment to particular infants they cared for and their sense of loss-following either their discharge or their death. Savage and Conrad (1992) refer to this vulnerability of the neonatal nurse as the likelihood to get "wounded." Following the philosopher Sally Gadow, they propose the strategy of "fellow-feeling" to prevent too close identification. Nurses should find a degree of emotional involvement in which they visualize the patient's or parent's emotion-like joy or suffering-while making sure not to experience it in the same way as they do. This "fellow-feeling" prevents "infectious identification" (p. 65). This approach enables nurses to find a balance between distance and attachment.

Yet, according to some health care scientists, the recognition of one's own fragility should also be considered as a positive that should not be evaded (Daniel, 1998; Malone, 2000; Spiers, 2000). Mutual vulnerability, they argue, generates compassionate engagement with a patient and contributes to bonding between nurse and patient. Daniel (1998) argues that the acknowledgment of a shared vulnerability is the key to authenticity. Acknowledging one's own vulnerability provides access to understanding a patient's experience. According to Daniel, nurses should take the opportunity to participate in vulnerability, rather than avoid it or protect themselves against it. As such, it is a vehicle for practicing authentic nursing and a way to celebrate humanness (p. 191). Thus vulnerability can result in a rich engagement with the patient (bond) or a walling-off (barrier), while sometimes a little of both is involved. This in turn requires careful balancing between engagement and dissociation. In an NICU, this is easier said than done. Treating NICU patients, which is far from an easy task, involves a delicate balance-not just physiologically, but also emotionally. As described next, in many cases, this balancing effectuates a relocation of risks and vulnerabilities. 
The Brittleness of Reliable Arrangements

The vulnerability of the patient population turns NICU practices into "high-3 work environments": they are packed with high technology, the work is characterized by high intensity and requires high reliability (Owen, Wackers, \& Béguin, 2009)..$^{5}$ A high-3 work environment comes with the potential of incidents that have drastic consequences on an everyday basis. Furthermore, its high level of interdependency and interrelations turns this complex socio-technical practice into quite a vulnerable system in itself. ${ }^{6}$ If NICU patients, parents, practitioners, their technology and interventions are vulnerable, the same applies to the organizational order of this practice. Moreover, even the safety measures designed to deal with these weak spots in the organizational structure are not always sufficient, as I will demonstrate in this section. Several safety measurements do not so much result in safety; rather, they are a matter of the relocation of risks that create their own vulnerabilities. Before describing safety measures in action in more detail, I will present some situations of the everyday organization of medical practice susceptible to mishaps.

\section{Risky Places and Tricky Times}

The intensive care of newborns requires the orchestration of a huge number of activities. Spinning a web of activities around and in the incubators involves a transition of information, people, and things. In the NICU, there are many moments of transitions, such as the handover of information and responsibility when the new shift of doctors and nurses arrive, or moving a patient to another incubator or ward. ${ }^{7}$ Throughout the day, there are multiple transitions of information, knowledge, equipment, and responsibilities between the different actors and organizations. These are the weak spots in the safety net and deserve attention. Another vulnerable part of the hospital organization pertains to the differences in expertise and experience among actors. This requires a balanced distribution of knowledge that the reality of care practices does not always allow. ${ }^{8}$ Take, for instance, the extreme instability of Daniel after his return from the cardiac surgery discussed earlier in this chapter. His instable condition requires the utmost concentration of all staff members involved. In these cases, the baby is not "just" a neonate, but also a cardiac patient. For such patients, other rules apply and other forms of reporting data are used. Therefore, doublechecking is called for when new data are added to the flow sheet. However, a post-surgical record, and a cardiac one in particular, consists of so many parameters that it is no longer possible to identify intervals between 
$\checkmark U$ practices into a technology, the reliability (Owen, $t$ comes with the an everyday basis. elations turns this iystem in itself. ${ }^{6}$ If and interventions er of this practice. I these weak spots , as I will demonso much result in 3 that create their $n$ action in more nization of medi-

$n$ of a huge numin the incubators n the NICU, there $f$ information and arrive, or moving he day, there are int, and responsihese are the weak rulnerable part of spertise and expeon of knowledge ake, for instance, le cardiac surgery quires the utmost ases, the baby is h patients, other herefore, doubleflow sheet. Howlar, consists of so intervals between the various checks. Also, the complexity of the monitoring of these kinds of complicated cases requires a certain level of specialized knowledge and experience that is not always available in every shift. In this respect, one of the NICU nurses commented:

The same staff members usually provide care to these children. This is why others, including me, do not gain any experience in treating them. So you can't expect us to do this straightaway. Just look at how this child is hooked up to everything. I can't do it. I just don't feel experienced enough to take over the responsibility for this baby.

Because highly complicated interventions, like open-heart surgery, are not daily routines, the staff has had little opportunity to gain experience with this type of cases. Although NICU staff has the expertise needed for this kind of treatment, it can be hard to distribute this expertise in a balanced way among the staff present at any given moment. The best staff members cannot be available around the clock, nor do they do all have the same amount of experience. This applies to both the nursing staff and the medical staff. Even if experienced physicians are normally present at night and during weekends to assist junior doctors to deal with unexpected complications, this does not always prevent near-incidents. Consider the following situation in the Dutch NICU:

Unexpectedly, the saturation level of Daniel's blood drops sharply (after his heart surgery). The attending has just left, and the resident is hesitant about what to do. Everyone is anxiously looking at the monitor. At that moment, the anesthesiologist and the cardiologist enter the NICU. They intervene instantly, and after some minutes the screen indicates that the saturation level improves again. The anesthesiologist is grumbling at everyone because upon entering she noticed that instead of acting they were all just watching the monitor.

Because of staff rotation and specific disciplinary differences between neonatology and cardiology, intensive monitoring, which is aimed at ruling out risks, becomes a source of uncertainty in its own way.

Aware of the vulnerability of their patient population, hospital practitioners and management try to preserve an adequate level of patient safety by initiating and implementing all kinds of safety measures. Next, I consider several of the initiatives that have been taken in health care over the last years, followed by some specific examples from the Dutch NICU.

\section{Safety Policy in Heaith Care}

In line with the safety policy in other high-risk industries, the strategy in health care is geared toward learning from mistakes. Accordingly, the 
reporting of errors is encouraged. These reporting systems make it possible for specific practices to aggregate data and recognize trends or patterns of adverse events and transfer back the lessons learned. ${ }^{9}$ Use of other information technology (TT), such as the computer physician order entry (CPOE), which provides the electronic infrastructure for doctor's orders on the treatment of patients, contributes to error reduction related to handwriting. Moreover, it has a built-in error-check for medication dose or treatment check. Similarly, the electronic patient record may have a central role in the improvement of patient safety. The ultimate aim is to replace the traditional individual report by an automated surveillance of clinical data (data-driven triggers) to detect and prevent events.

In addition to the systematization of health care, new views stress the cultivation of a safety culture (Aspden et al., 2004). A culture of safety entails alertness, openness, transparency, learning, and improving, as well as the recognition of inevitable human limitations such as those tied to fatigue, vigilance, and memory (Bargian, 2006). Such culture also involves professional education, management involvement, open communication lines, leadership, and teamwork. Safety studies in aviation, for example, have shown the importance of teamwork. Crew resource management training teaches pilots how to deal with high levels of stress in complex flight situations. Likewise, hospitals organize teamwork training for staff members working in critical care, such as intensive care units, operating rooms, and emergency rooms (Helmreich \& Schaefer, 1994; Randell, 2003). ${ }^{10}$ By means of collaborative learning, the health care sector tries to bring about "breakthrough improvements" through diffusion of best practices (Institute for Healthcare Improvement, 2003). In this way, it tries to spread evidencebased knowledge over as many as possible health care organizations.

The development of blame-free reporting systems, electronic patient records, crew resource management training, and the diffusion of best practices and safety cultures are considered essential for the improvement of patient safety. However, the implementation of these safety measures has met with problems. The reporting system, for example, is hindered by a "culture of blame." The fear of being blamed and being held responsible or being subjected to disciplinary measures inhibits staff members to participate in reporting incidents. Many efforts are geared to transforming the "blame culture" into a "safety culture." It is recognized that feedback is crucial in motivating physicians and nurses to report errors. Additional measures are taken to overcome the blame culture's detrimental effects. Enr inctance rennrtino forms are designed to guarantee the anonym- 
ms make it possible ends or patterns of se of other informaorder entry (CPOE), :or's orders on the elated to handwritation dose or treat$y$ have a central role im is to replace the ince of clinical data

lew views stress the ture of safety entails Jving, as well as the hose tied to fatigue, llso involves profesImmunication lines, , for example, have lanagement training complex flight situig for staff members perating rooms, and :11, 2003)..$^{10}$ By means , bring about "breakactices (Institute for to spread evidenceorganizations.

s, electronic patient he diffusion of best for the improvement hese safety measures sxample, is hindered id being held responbits staff members to sared to transforming ignized that feedback ort errors. Additional $s$ detrimental effects. irantee the anonymshow staff members that reporting is a necessary condition for learning from mistakes. Waring (2005) argues, however, it is naïve to think that it is merely a matter of transforming a "blame culture" into a "just culture." Other cultural aspects play a role as well in this matter. For instance, many actors in medical culture will consider errors an inevitable part of doing medical work, but from this perspective incident reporting has little significance. Also, collegiality and a deep-rooted aversion to administrative work (which also comes with more managerial interference) do not contribute to incident reporting among doctors, according to Waring. In addition, the steep hierarchical structure of medicine makes it more difficult to acknowledge the effects of stress and fatigue or to admit mistakes (Randell, 2003). Even when the reporting rate is high, clinicians responsible for the root-cause analysis of the reports have great difficulty to derive organizational-managerial generalizations from, sometimes contradictory, details of the described situated action (Iedema et al., 2006). Furthermore, they expect their recommendations to result in general rules that will improve the health care system.

\section{Safety Measures in Action}

Some safety measures may prevent specific errors but create unsafe situations of another kind instead. Take, for instance, the double-check of medication-one of the most applied safety measures in medical practice. In NICUs, medication is prescribed by the attending physician, but it is prepared by nurses. The nurses always check if they prepared the right substance and dose for the correct patient at the right time. In addition, they will ask a colleague to check their preparations. Because most NICU patients receive their medications around the same time, one will find the nurses working side by side at the medication desk, where they can help each other to double-check their work. If not, nurses need to ask a colleague who is busy taking care of one of the babies in another way. But in both situations the request for a double check may affect their colleague's level of concentration. For instance, a nurse at work next to the incubator may forget that he or she has changed the settings of the respirator while performing a stressful procedure (such as suctioning). In this case, the double check does not resolve the potential for mistakes, but only relocates the vulnerability of the organizational arrangements. It moves vulnerability away from the medication provision to the monitoring activity. ${ }^{11}$

Similarly, the application of IT tools such as the CPOE is not without risks. Although many consider IT the magic bullet for all sorts of problems, it can also introduce other types of error into the care process. Consider, for example, a juxtaposition error where clinicians order the wrong tests 
due to the screen design or incidents because the computerized medication system does not allow alternative practices that are sometimes needed in cases of urgency (Ash, Berg, \& Coiera, 2004). Dealing with the vulnerable parts in the organization is hardly an easy task. Some measures seem to solve one problem, but they create another one. Strengthening the safety net in one corner can be at the cost of the robustness of another part. In this case, vulnerability is not solved but relocated.

Relocation of vulnerability is also visible in the efforts of the staff at the Dutch NICU to deal with disturbances during medical rounds. In the practical order of this NICU, there used to be a condensation of different lines of activity around noon, with doctors doing their daily medical round-checking the condition of the babies and discussing treatment policies-while nurses would provide information about the babies and their parents. However, it was quite common at that time of the day to see half the nursing staff busy organizing their lunch break; in their minds, the nurses were already on their way to the cafeteria while finishing a final check of a baby.

Even when the nurses were normally available, however, there was no guarantee that everything went well during medical rounds. For example, it was common for the nurse in charge of a baby to write down the doctor's orders on the nursing form. Frequently, though, the neonatologists not only would tell nurses about a child's new or changed doses of medication, but they would also change the settings of the machines in the meantime. Nurses busy recording information did not always notice this, of course. Or doctors would ask a nurse to change the setting, upon which she or he stopped entering information on the nursing form while the doctors continued to discuss the next steps in the treatment trajectory of the child at hand. Meanwhile, other nurses might ask the fellow or attending neonatologist for advice on small but urgent matters, thus interrupting the medical round. On top of this, there is the usual NICU noise, such as ringing phones and sounding alarm bells, which sometimes makes it difficult for everyone to hear what is said. As one can imagine, this multitasking-changing settings, listening to the discussion, informing doctors, writing notes, and so on-easily led to errors and mistakes on the part of the nurses.

After realizing the risks of this organizational weak spot, doctors changed the setup of medical rounds. They moved the daily rounds to the ward's meeting room. Now the nurses took turns in the discussion, and their presence determined which baby was discussed at what moment. In this way, nurses and doctors were not distracted anymore. However, in the meantime, there were no doctors on the NICU, while medical rounds took twice 
2nts because the computerized medication re practices that are sometimes needed in 'oiera, 2004). Dealing with the vulnerable dly an easy task. Some measures seem to ate another one. Strengthening the safety cost of the robustness of another part. In ved but relocated.

; also visible in the efforts of the staff at isturbances during medical rounds. In the lere used to be a condensation of differon, with doctors doing their daily mediion of the babies and discussing treatment rovide information about the babies and uite common at that time of the day to see anizing their lunch break; in their minds, - way to the cafeteria while finishing a final

normally available, however, there was no : well during medical rounds. For example, charge of a baby to write down the doctor's requently, though, the neonatologists not 'hild's new or changed doses of medication, : settings of the machines in the meantime. tion did not always notice this, of course. J change the setting, upon which she or he on the nursing form while the doctors con$s$ in the treatment trajectory of the child at might ask the fellow or attending neonatolgent matters, thus interrupting the medical he usual NICU noise, such as ringing phones :h sometimes makes it difficult for everyone $n$ imagine, this multitasking - changing setin, informing doctors, writing notes, and so stakes on the part of the nurses.

is organizational weak spot, doctors changed They moved the daily rounds to the ward's ; took turns in the discussion, and their preswas discussed at what moment. In this way, listracted anymore. However, in the meani the NICU, while medical rounds took twice as long because doctors had to wait for nurses, who first had to finish a task or hand over their responsibilities to a colleague. During these handover moments, the medical staff cannot do anything but wait for the nurse involved to join them. But also the doctors themselves took more time to discuss the children and their medical conditions, also because medical rounds were increasingly devoted to training new staff. This has led to a considerable reduction of the time the doctors were in fact available on the NICU. Rather than solving the initial problem, then, in the new configuration, it was largely relocated to the nursing practice. At noon, vulnerability was now ensconced in the density of available medical expertise on the floor.

Besides a spatial relocation of doctors and nurses and their attention to other places, some safety measures produced a temporal relocation. For example, the preparation of medication in the NICU was taken over by a special satellite of the pharmacy that was located across from the NICU. Due to this new service, the level of medication errors dropped significantly during weekdays. However, on weekends, this satellite was unstaffed, and the nurses had to prepare the medication themselves, including those that were not prepackaged. Because they had become accustomed to using the satellite service of the pharmacy, the nursing staff were unused to preparing medication, causing them to lose their skill and knowledge of this task, which in turn led to a growing number of medication errors on the weekends.

Examples such as these make clear that it is not so much the use of specific techniques that lead to the reduction of an organization's vulnerability, but rather the (organizational) contexts, including the overall organizational system, in which they are put to use. Despite all the efforts made to improve patient safety, it is clear to everyone involved that improving patient safety is hardly a straightforward matter. The level of complexity in health care practices is such that unintended, collateral effects of safety measures can be neither predicted nor prevented. Moreover, some of the measures used to cope with the NICU's vulnerabilities actually contribute to an unsafe practice.

\section{Concluding Remarks}

The preceding sections of this chapter illustrate the shapes that vulnerability can take in critical care practices. It can be located on the level of the actors and their actions, as well as on the level of the specific arrangements that guide their work. Analysis of complex situations contributes to our 
understanding of how the distinct vulnerabilities complicate the work in a critical care practice like the NICU in several ways.

First, as the empirical data in this chapter demonstrate, vulnerability is not an intrinsic property of human beings or a specific situation, but an emergent property. At first sight, it seems clear to doctors and nurses how act safely and which path of action they need to avoid. Vulnerability appears to be fixed to specific parts and processes of the baby's body, the work, and the health care organization, as if it is an inherent property of these aspects and processes. However, the empirical data show this to be an inadequate depiction of NICU practice. Vulnerability emerges only in a specific context, as an outcome of specific circumstanced, but also between ability is also about relations: between the baby's body, or between the morning the different vital functions in the relationships need to be tightshift and the evening shift. Some of these relationships nuch as insertion ened, such as that of mother and child, while others, need to be disconof an intravenous (IV) line and bloodstream infecefully, while others comnected. Some of these

Second, vulnerability comes in many ways. Infectious identification, mmature lungs, and parental anxiety are among the many kinds of vulnerability that inhabit the NICU practice. These multiple vulnerabilities are redistributed over time and place but never leave the NICU practice. As the the compensated for by the suggestion of control that cize medical action, the involved actors try to find a way tends to characterize medical actions stizing conditions and tightening or loos to deal with its presence by stability is an integral component of critical care ening linkages. But vulnerability is an in the staff is at trying to avoid it. practices, no matter how successful the staff is at trying to avoid ifferent levels: namely,

Third, in NICU practice, vulnerabilty is found the levels of actors, actions, and arrange NICU patients, the extent to which parbility of the vital functions of the Nicu pot having a healthy child, or ents can cope with the emotional burden of not having itication. But it not the ability of caregivers to withstand infectious identes, and arrangements only people who are vulnerable; actions, objects, mistortion.

also can be fragile or susceptible to diversion and setting-like the patients,

Fourth, the actors who live and work in this separate; they interact, as do their their parents, and caregivers--are not separanizational arrangements. This planned and unplanned actions and the level and locus of vulnerability. dynamic contributes in its own way to the level and coxist in the NICU pracAs we have seen, different kinds of vulnerabilites coexist in the certain measures tice, and in some cases, they even compete. Moreover, cer 
vulnerabilities complicate the work in a in several ways.

lis chapter demonstrate, vulnerability is in beings or a specific situation, but an seems clear to doctors and nurses how ction they need to avoid. Vulnerability ts and processes of the baby's body, the cation, as if it is an inherent property of ever, the empirical data show this to be practice. Vulnerability emerges only in a f specific circumstances. As such, vulnereen the actors involved, but also between le baby's body, or between the morning $a$ of these relationships need to be tightid child, while others, such as insertion loodstream infection, need to be disconities coexist peacefully, while others com-

in many ways. Infectious identification, Ixiety are among the many kinds of vul' practice. These multiple vulnerabilities are but never leave the NICU practice. As the asated for by the suggestion of control that tion, the involved actors try to find a way rilizing conditions and tightening or loos$y$ is an integral component of critical care sful the staff is at trying to avoid it. erability is found on different levels: namely, 1 arrangements. It concerns the level of stahe NICU patients, the extent to which paral burden of not having a healthy child, or stand infectious identification. But it is not e; actions, objects, rules, and arrangements e to diversion and distortion.

and work in this setting-like the patients, are not separate; they interact, as do their $s$ and the organizational arrangements. This I way to the level and locus of vulnerability. $s$ of vulnerabilities coexist in the NICU praceven compete. Moreover, certain measures and strategies to strengthen bodies and practices relocate vulnerability instead of diminishing it. Vulnerable situations are moved to another location or to another moment, but they are not removed altogether.

Fifth, vulnerability is a form of potential distortion and disconnection of parts and processes. Surviving premature birth or providing care and treatment in a critical care context is a matter of attaining and solidifying a stable, healthy state. ${ }^{12}$ For instance, it is about the delicate balance of the input and output of fluid supply in the baby's body, or about the choice between doing blood tests for data collection or providing comfort instead. It is also about the necessity to discuss treatment policies and availability of doctors on the ward, and about the number of experienced versus less experienced personnel. These issues have to be balanced, but they are in fact unstable, so continuous adaptation is required.

Finally, we have to be careful not to consider vulnerability as purely a negative matter. Vulnerability can prompt processes of awareness and alertness. The vulnerability of the children, for example, triggers the alertness of practitioners and the improvement of the sensitivity of machines and the refinement of their settings. The delicacy of the balance between the efficacy and safety of medical interventions also contributes to the level of situational awareness and the significance of patient safety measures. In other words, vulnerability also contributes to making the NICU a safe place to be. Or, in the words of one of the practitioners:

It is the vulnerability of the baby's body that triggers a high level of vigilance. When you take care of a patient who is as fragile as a NICU baby, it implies that you cannot afford any mistakes. You have to be very careful in everything you do. The staff on this ward is fully aware of the vulnerability of their patient population, and that keeps them highly alert. The vulnerability of NICU babies is appealing to the staff's responsibilities, so to speak.

\section{Notes}

1. Some parts of this chapter are based on my study of medical and ethical uncertainty in the NICU, as published in Mesman (2008), and are reproduced with the permission of Palgrave Macmillan.

2. My argument here relies on data that I collected during ethnographic research in a Dutch and an American NICU in the 1990s and the years 2008 to 2010.

3. The treatment of criticaly ill newborns often gives rise to moral dilemmas. Several ethnographic studies provide insights into the complexity of these kinds of problems. See, for example, Anspach (1993), Frohock (1986), Guillemin and Holmstrom 
(1986), Lantos and Meadow (2006), and Mesman (2008). Although highly important, the issue of moral dilemmas is not the focus of this chapter.

4. For more on this topic, see Mesman (2008).

5. High-3 work environments can also be found in, for instance, the aviation industry, the chemical industry, and nuclear power plants.

6. The way that I refer here to the vulnerability of the health care organization should not be confused with the "vulnerable system syndrome" as developed by Reason, Carthey, and de Leval (2001), which refers to a specific cluster of "organisational pathologies."

7. Handover moments have been a topic of investigation for many researchers (e.g., Broekhuis \& Veldkamp, 2007; Iedema, 2007; Iedema et al., 2009; Kerr, 2002; Manias $\&$ Street, 2000).

8. See chapter 8 of this volume, "Resilience: Contingency, Complexity, and Practice" for a discussion of resilience in these kinds of circumstances.

9. Cf. Aspden et al. (2004, 2007), Kohn et al. (2000).

10. The relevancy of collaboration is not limited to the level of health care practitioners; collaboration between health care practices is considered a crucial building block for patient safety as well.

11. In addition, the double-check system does not prevent the situation where a medication is not handed out at all.

12. Sometimes children are referred to as stable in their being ill; their sickness may be a consistent condition, if not a desired one. 


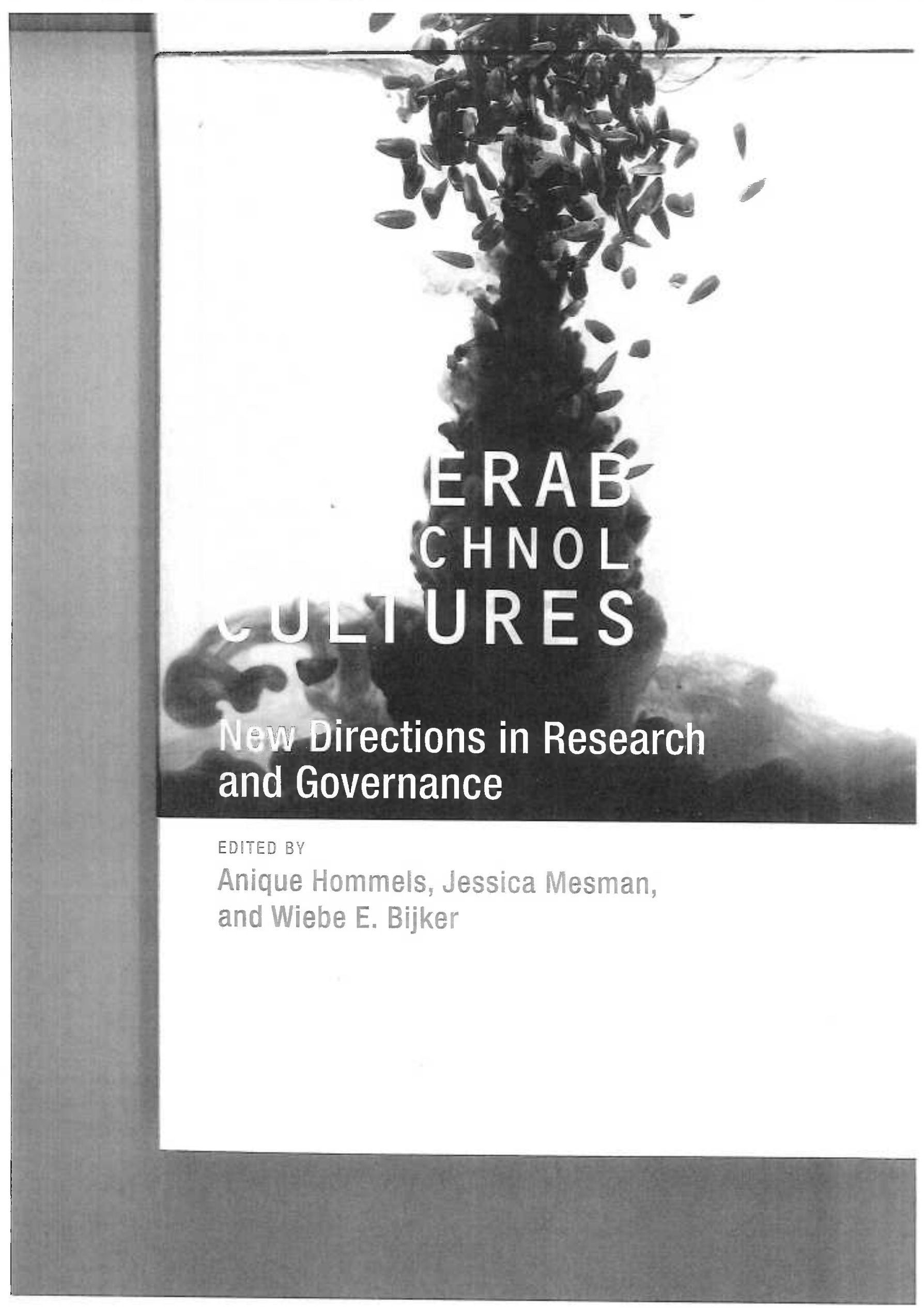

